\title{
Burr Holes Revascularization in Three Pediatric Cases of Moyamoya Syndrome: Easy Choice or Insidious Trap? Case Series and Review
}

\begin{abstract}
Introduction: Moyamoya disease is a steno-occlusive cerebrovascular disease of unknown etiology involving the terminal portion of the internal carotid artery and the proximal portions of the anterior and middle cerebral arteries with associated collateral vascular network. When the vascular pattern is associated with a particular condition (e.g., Type 1 neurofibromatosis, Down syndrome), it is defined as moyamoya syndrome (MMS) (or quasi-moyamoya). Among different indirect bypass techniques used to prevent ischemic injury by increasing collateral blood flow to hypoperfused areas of the cortex, multiple burr holes technique is an easy and diffuse indirect revascularization approach in the treatment of moyamoya. Discussion: While the effectiveness in patients with moyamoya disease was demonstrated, its role in MMS remains uncertain. In this study, we describe surgical and diagnostic implications in three pediatric cases of moyamoya sydrome unsuccessfully treated with multiple cranial burr hole technique. A critical review of the literature about the use of the surgical indirect revascularization techniques in pediatric patients was also reported.
\end{abstract}

Keywords: Burr holes, bypass, indirect, moyamoya, revascularization

\section{Introduction}

Moyamoya disease is an idiopathic steno-occlusive cerebrovascular disease of unknown etiology, defined by a progressive steno-occlusive modification of the distal internal carotid artery (ICA) and its proximal branches that evolve in the development of an extensive collateral basal vascular network.

If the typical vascular pattern is associated with an underlying systemic condition (e.g., Type 1 neurofibromatosis (NF1), Down syndrome extracorporeal circulation), the illness is named moyamoya syndrome (MMS) or quasi-moyamoya.

A recent Japanese epidemiological analysis revealed an incidence of $0.11 / 100.000 /$ year $(0.11)$ and a prevalence of $0.34 / 100.000$ for MMS and an incidence of $1.13 / 100000$ and a prevalence of $5.22 / 100000$ for moyamoya disease. ${ }^{[1]}$

Moyamoya patients usually present after ischemic symptoms in juvenile age and after cerebral hemorrhage in adult age, with a difference in onset age among different entities. No medical treatment has been proven to be effective neither in preventing

This is an open access journal, and articles are distributed under the terms of the Creative Commons Attribution-NonCommercialShareAlike 4.0 License, which allows others to remix, tweak, and build upon the work non-commercially, as long as appropriate credit is given and the new creations are licensed under the identical terms.

For reprints contact: reprints@medknow.com these events nor in stopping the progressive steno-occlusive transformation. The aim of the surgical treatment is to improve brain perfusion in ischemic or nearly ischemic territory. This has led to the development of multiple surgical strategies which can be classified into two categories: (1) direct and (2) indirect surgical revascularization techniques. The direct revascularization is performed by creating a direct vascular anastomosis between a branch of the superficial temporal and the middle cerebral or anterior cerebral artery (ACA). Indirect revascularization techniques enhance vascularization of hypoperfused cerebral territories by opposing different vascularized tissue (superior temporal artery itself, the omentum, the temporalis muscle, the periosteum, or combination of them) over the cortical surface of the brain.

The burr holes technique is an indirect revascularization procedure first described by Endo in 1984. The procedure consists in drilling multiple holes through the skull to stimulate angiogenesis through the holes, from branches of the external carotid artery or the middle meningeal artery (MMA). While the burr hole technique demonstrated

How to cite this article: Pacetti M, Tortora $D$, Fiaschi P, Consales A, Piatelli G, Ravegnani M, et al. Burr holes revascularization in three pediatric cases of moyamoya syndrome: Easy choice or insidious trap? Case series and review. Asian J Neurosurg 2018;13:769-73.

\section{Mattia Pacetti, Domenico Tortora ${ }^{1}$, Pietro Fiaschi, Alessandro Consales, Gianluca Piatelli, Marcello Ravegnani, Armando Cama, Marco Pavanello}

Department of Neurosurgery, ${ }^{1}$ Department of Neuroradiology, Istituto Giannina Gaslini Children's Hospital, Genova, Italy
Address for correspondence: Dr. Pacetti Mattia, Istituto Giannina Gaslini Children's Hospital, Via Gerolamo Gaslini, 5, 16148 Genova, Italy. E-mail:mattiapacetti@ gmail.com

Access this article online Website: www.asianjns.org DOI: 10.4103/ajns.AJNS_155_16 Quick Response Code:

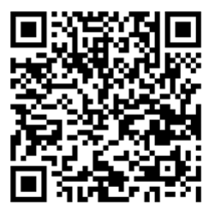


good effectiveness in patients with moyamoya disease, ${ }^{[2]}$ the outcome after the burr holes procedure in patients with MMS remains uncertain. ${ }^{[3]}$

We present three cases of pediatric patients with MMS unsuccessfully treated with multiple cranial burr hole $(\mathrm{MCBH})$ technique that required a second surgical indirect revascularization. Furthermore, we reported the surgical and diagnostic implications of a second surgical procedure performed after the multiple burr hole revascularization.

\section{Illustrative Cases}

\section{Patient 1}

The patient, a 6-year-old female, presented at our institution with headache as major complaint. Her history began at the age of one when NF1 was clinically diagnosed. Brain magnetic resonance imaging (MRI) revealed bilateral focal area of T2-hyperintensity and T1-hypointensity (unidentified bright objects) in the basal ganglia and in the cerebellar white matter. Arterial MRI angiography showed the narrowing of left ICA and middle cerebral arteries (MCA) with surrounded neoangiogenesis and omolateral MMS was diagnosed. After 12 months from clinical onset, a surgical revascularization with burr holes technique was performed in the left parietal and temporal bones with resolution of headache. Antiplatelets therapy was administrated.

After a normal postsurgical period of 11 months, the patient presented again with omolateral (left) headache and right arm paresthesia. Digital subtractive angiography and MRI angiography showed worsening of the moyamoya stage (Suzuki Stage 3) and prominent leptomeningeal hyperintensities along cortical sulci of the left hemisphere was seen in the MRI fluid-attenuation inversion recovery sequence (total ivy sign score 5) [Figure $1 \mathrm{~b}$-c]. Single-photon emission computed tomography (SPECT) perfusion demonstrated brain hypoperfusion in the left frontal, temporal, and parietal lobes and the bone three-dimensional (3D) computed tomography (CT) scan revealed nearly complete closure of the left burr holes [Figure 1a]. Consequently, a new surgical intervention was indicated, using a left indirect revascularization technique (encephalo-duro-arterio-myoperiosteo-synangiosis).

At the last follow-up after 2 years from the last surgical procedure, neurological symptoms improved with regression of the headache. Brain MRI revealed reduction of the ivy signs and SPECT examination demonstrated increased perfusion in the left hemisphere [Figure 2].

\section{Patient 2}

The patient, a 9-year-old male, was admitted to another hospital for visual function impairment. The brain MRI revealed a solid enhancing tumor with multiple cystic

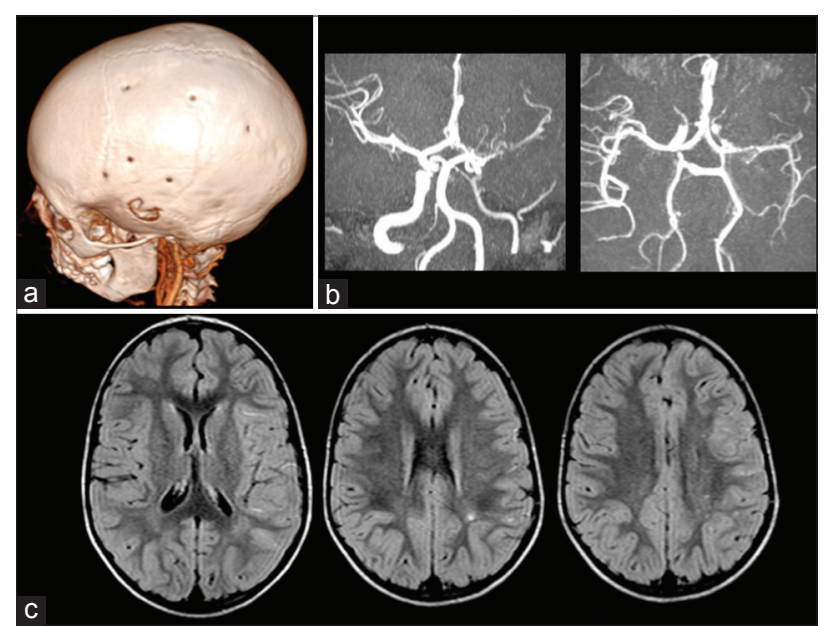

Figure 1: Case 1 - A 6-year-old female with Type 1 neurofibromatoris and moyamoya, unsuccesfully treated with multiple burr holes technique, requires a second revascularization procedure (encephalo-duro-arterio-synangiosis/ encephalo-duro-artero-mio-periosteo-synangiosis). Preoperative evaluation performed before the second intervention. (a) Cerebral computed tomography scan three-dimensional volume-rendering shows the shrinkage of the multiple burr holes in the left parietal and temporal bones. (b) Coronal and axial maximum intensity projection-reformatted magnetic resonance imaging angiography show narrowing of both the left internal carotid artery and ipsilateral middle cerebral artery. (c) Axial fluid-attenuation inversion recovery images show leptomeningeal hyperintensities along cortical sulci in the left frontal and parietal lobes ("ivy sign")

components involving the hypothalamus, chiasm, and optic radiations [Figure 3a-b]. The patient underwent an endoscopic resection of tumor cyst. The final diagnosis of ganglioglioma of the hypothalamic-chiasmatic region was made histopathologically. After about 24 months from radiotherapy (54 Gy of total radiation dose), the patient progressively developed a deep collateral network, associated with steno-occlusive transformation of the left sovra-clinoid ICA and left middle carotid artery as seen on arterial MRI angiography [Figure 3c-d]. SPECT perfusion revealed bilateral frontal and parietal hypoperfusion. The patient cognitive score was measured and a mild impairment was noted. Consequently, the diagnosis of MMS was made. After 3 months, a surgical revascularization with burr holes technique was performed bilaterally in the frontal, parietal, and temporal bones.

After 10-month follow-up, the left side paresthesia in perioral area and upper limb was noticed, and it was confirmed by a less evident perfusion in right pre- and post-central gyrus.

At the last follow-up control performed in our hospital at 17 years old, no improvement in brain perfusion was revealed at the SPECT examination. Therefore, a new procedure of indirect revascularization including also the ACA territories was considered to prevent a further cognitive impairment of the patient.

\section{Patient 3}

The patient, a 6-year-old female, presented impairment of the visual function and headache. The brain MRI, performed 


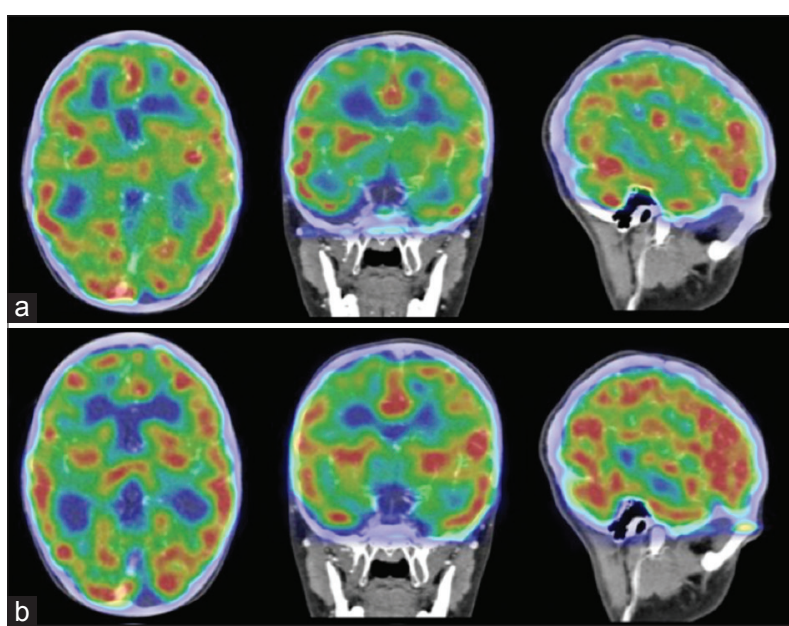

Figure 2: Case 1 - Brain single-photon emission computed tomography perfusion images fused on the cerebral angio-computed tomography scan performed after 1 year from the first revascularization procedure (multiple burr holes) (a) and 2 years after the second indirect revascularization (encephalo-duro-arterio-synangiosis/encephalo-duroartero-mio-periosteo-synangiosis) (b). Left frontal and parietal lobes remain hypoperfused after burr holes revascularization and recovers after encephalo-duro-arterio-synangiosis/encephalo-duro-artero-mio-periosteosynangiosis procedure

in another hospital, revealed a suprasellar/parasellar tumor with an enhancing solid portion with associated multiple cystic structures. The lesion was treated with surgical debulking and subsequent adjuvant chemotherapy and radiotherapy (Society of Paediatric Oncology low-grade glioma 2004 protocol). The final diagnosis of pilomyxoid astrocytoma of the hypothalamic-chiasmatic region was made histopathologically. After about 2 years, the patient presented headache and paresthesia in the right upper limb. The brain MRI revealed some new small areas of T2-hyperintensities in the left perirolandic region suspected for ischemic vascular lesions. The diagnosis of MMS was done basing on the arterial MRI angiography which detected the narrowing of the left ICA and the occlusion of the ipsilateral MCA [Figure 4a-b].

After about 3 months from the onset of symptoms, the patient, admitted at another institution, underwent an indirect surgical revascularization with burr holes technique in the left frontal, parietal, and temporal bones.

At the follow-up controls performed every 6 months in our hospital, the neurologic symptoms persisted and the patient showed a rapid impairment of cognitive functions, showing a loss of 10 points at the full-scale intelligence quotient (wechsler intelligence scale for children V). MRI brain perfusion confirmed the hypoperfusion of the left MCA territory with minimal improvement only in the left temporal lobe. Therefore, a new indirect revascularization was planned to guarantee an adequate perfusion. Before the surgery, it was necessary to determine which burr holes developed angiogenesis and which was the relationship between the successful burr holes and the brain areas that remained hypoperfused. A CT scan [Figure 4c] with

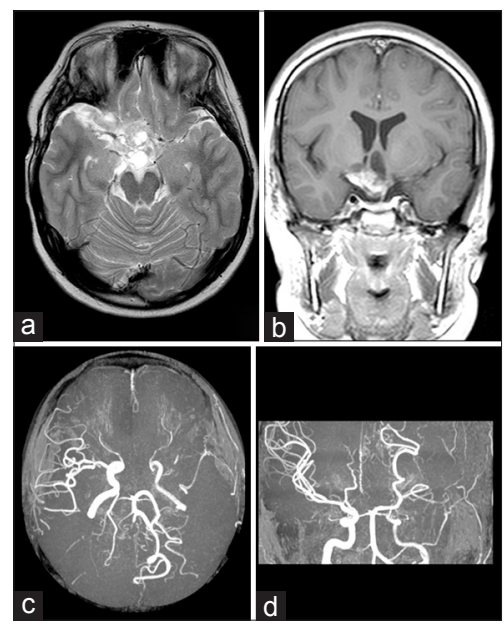

Figure 3: Case 2 - A 9-year-old male with suprasellar ganglioglioma and moyamoya syndrome, unsuccessfully treated with multiple burr holes technique. ( $a$ and b) Axial turbo spin-echo T2-weighted and coronal postgadolinium standard error T1-weighted images show the residual sellar-suprasellar ganglioglioma with a solid enhancing portion and pseudocystic parts. (c and d) Axial and coronal maximum intensity projection-reformatted magnetic resonance imaging angiography show narrowing of the left internal carotid artery and occlusion of the ipsilateral middle cerebral artery

CT angiography was performed to evaluate the anatomy of the angiogenetic vessels and a MRI perfusion study, with arterial spin labeling technique, was used to identify the hypoperfused brain areas [Figure 4d]. The line of the surgical craniotomy was established on the fusion of two different examinations preserving the portion of the skull containing the burr holes with new angiogenesis [Figure 5].

\section{Discussion}

Surgical revascularization is the mainstay of the treatment of moyamoya disease and syndrome.

Among different types of procedure, burr hole surgery demonstrated effectiveness in revascularizing ischemic brain tissue in the pediatric and adult patients with moyamoya disease. Data suggest that all surgical treatments, when performed at high-volume centers on carefully selected patients, yield similar excellent results regardless of the surgical approach. Indeed, to date, there is no randomized trial assessing the superiority of any surgical technique over any other. ${ }^{[2]}$

Endo et al., 1989, ${ }^{[4]}$ after the incidental discovery of a certain revascularization potential from a frontal burr holes placed for external ventricular drainage, initially proposed to use $\mathrm{MCBH}$ in combination with encephalo-myosinangiosis presenting a series of five pediatric cases. The authors concluded that burr hole surgery was a beneficial complement to standard revascularization surgeries for juvenile cases of myotonic muscular dystrophy (MMD). Suzuki et al. ${ }^{[5]}$ also evaluated the use of burr holes in combination with other surgical techniques in a series of pediatric MMD patients. They proposed the use of a single frontal burr hole technique combined with the 


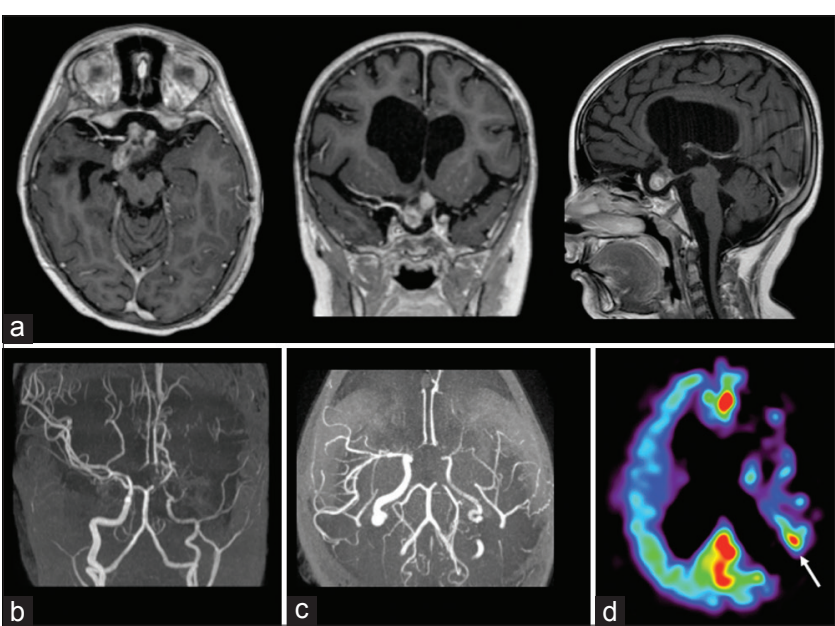

Figure 4: Case 3 - A 6-year-old female with impairment of the visual function and headache. (a) Axial and coronal potgadolinium standard error T1-weighted images show suprasellar lesion with solid enhancing portion. (b) Coronal and axial maximum intensity projection-reformatted magnetic resonance imaging angiography show narrowing of both the left internal carotid artery and ipsilateral middle cerebral artery. (c) Cerebral computed tomography scan three-dimensional volume-rendering shows multiple burr holes performed in the left parietal and temporal bones. Some holes in parietal bones are shrinking. (d) Arterial spin labeling cerebral blood flow map shows persistent hypoperfusion in the left middle cerebral artery territory. White arrow indicates a transit artifact suggesting a delayed blood flow

superficial temporal artery MCA bypass in children older than 5 years while in younger patients, they suggested to perform encephalo-duro-arterio-synangiosis combined with burr holes. A satisfactory outcome was also described by Robertson et al. ${ }^{[6]}$ using pial synangiosis and a single frontal burr hole. Dusick et al. published their results regarding the combination of the burr holes with different surgical techniques. ${ }^{[7]}$ Another indication for combining one frontal burr hole with another indirect revascularization technique (pialsynangiosis) came from Scott et al., ${ }^{[8]}$ focusing on a group of syndromic moyamoya patients only, describing good clinical and radiological results.

In more recent times, different authors described the use of MCBHs alone, starting from 1996, when Kawaguchi et al. reported a series of $10 \mathrm{MMD}$ adult patients, treated with a variable number of burr holes. ${ }^{[9]}$ Results of this first series showed a good revascularization, mainly from collateral derived from MMA and subsequently, superficial temporal artery. Despite these good results, 10 years had passed before another publication concerning the use of $\mathrm{MCBH}$ alone appeared. In 2006, Sainte-Rose et al. reported a series of 14 pediatric MMD patients treated using a larger number of burr holes aiming at an extensive revascularization from occipital to anterior territory. Good results were objectived by postoperative angiography and in five cases only, by perfusion MRI performed after 3 months. ${ }^{[10]}$ Oliveira et al. carried out similar procedure in a series of seven pediatric MMD patients, presenting good angiographic outcome showing an extensive collateralization by external carotid artery collaterals. ${ }^{[1]} \mathrm{A}$ case report by Calviere et al.

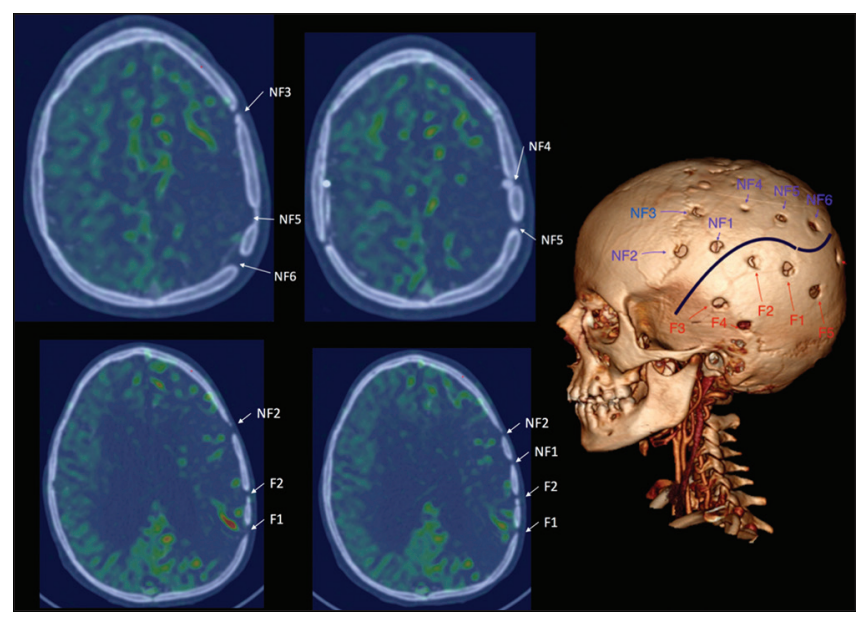

Figure 5: Case 3 - Arterial spin labeling perfusion images fused on the cerebral angio-computed tomography scan performed after almost 1 year from the first revascularization procedure (multiple burr holes). "NF" indicates holes which did not develop angiogenesis. "F" indicates hole which developed angiogenesis. The site of craniotomy was decided on the fused images preserving the portion of the skull containing burr holes with new angiogenesis

presented a revascularization procedure with bilateral burr hole surgery performed in a 39-year-old woman, which resulted in complete regression of cognitive impairment. Improvement in cognitive function correlated with the development of transdural collaterals on angiography and improvement in cerebral perfusion on MRI. This case suggested a relationship between cognitive dysfunction and cerebral hypoperfusion in MMD. In the opinion of the authors, cognitive impairment may be potentially reversible after burr hole surgery and cerebral perfusion improvement. ${ }^{[12]}$ Another case was published by Kapu et al. who described a multiple burr hole surgery procedure (five frontal, temporal, and parietal holes per side) as a treatment for a pediatric symptomatic bilateral MMD patient. ${ }^{[13]}$ Clinical outcome was good but with one transient ischemic attack after 6 months. Angiogram showed revascularization around burr holes.

None of the cited article refers about the possibility of a second operation, after the radiological and clinical evidences of failure of the MCBH procedure. As described above, we managed three pediatric cases of burr holes technique revascularization failure where we proposed a second surgery, using another indirect technique. Two different types of issues should be considered: radiological and surgical. First, the needing of second surgery after a $\mathrm{MCBH}$ procedure makes the presurgical planning much complex, to understand the relationship between the position of the burr hole and the effective cortical hypoperfusion notch. To better visualize the effective situation, we used a fusion of 3D CT bone scan and perfusion MRI [Figure 5]. Further, skin incisions and craniotomy were planned to avoid the disconnection of the revascularization already occurred in some of the burr holes (see figure legend for details), avoiding the theoretical risk of acute ischemia. 
Every time, a first surgery or reoperation is planned, several factor should be considered before to decide the best revascularization options, for example, the presence of cortical atrophy, with an expansion of the subdural space that could decrease the development of neovascularization, ${ }^{[14,15]}$ or the presence and the caliber of superficial temporal artery.

We think that our experience could confirm, as reported by a literature analysis, that the role and the type of optimal surgical revascularization technique for pediatric MMS remain controversial. Furthermore, data from previous medical literature suggest that surgical revascularization is a safe intervention for pediatric MMS and most of the treated patients derive some symptomatic benefit, but the poor data on selection criteria and more global outcome measures mean that even the long-term impact of surgical revascularization on natural history remains uncertain. ${ }^{[3,16,17]}$

\section{Conclusions}

Considering data from the literature, we could state that, according to our experience, a combined indirect approach should be preferred, especially in syndromic moyamoya patients. MCBHs technique has a great importance and its indications should be known by every surgeon dealing with MMS and disease. MCBH is indeed an easy procedure that could lead to get a good degree of neoangiogenesis and it is useful to try to tailor revascularization, especially in most frontal cortical territories. Nonetheless, we advise that it should not be considered alone as the standard initial operation for every moyamoya patient since, as reported, its radiological and/or clinical outcome could be less than optimal, especially in syndromic moyamoya patients. To avoid the multiple diagnostic and reoperative surgical caveats that we already described, we advise that, if feasible, other indirect or combined procedures should be chosen.

\section{Financial support and sponsorship}

Nil.

\section{Conflicts of interest}

There are no conflicts of interest.

\section{References}

1. Baba T, Houkin K, Kuroda S. Novel epidemiological features of moyamoya disease. J Neurol Neurosurg Psychiatry 2008;79:900-4.

2. McLaughlin N, Martin NA. Effectiveness of burr holes for indirect revascularization in patients with moyamoya disease $-\mathrm{A}$ review of the literature. World Neurosurg 2014;81:91-8.
3. Fung LW, Thompson D, Ganesan V. Revascularisation surgery for paediatric moyamoya: A review of the literature. Childs Nerv Syst 2005;21:358-64.

4. Endo M, Kawano N, Miyaska Y, Yada K. Cranial burr hole for revascularization in moyamoya disease. J Neurosurg 1989;71:180-5.

5. Suzuki Y, Negoro M, Shibuya M, Yoshida J, Negoro T, Watanabe K. Surgical treatment for pediatric moyamoya disease: Use of the superficial temporal artery for both areas supplied by the anterior and middle cerebral arteries. Neurosurgery 1997;40:324-9.

6. Robertson RL, Burrows PE, Barnes PD, Robson CD, Poussaint TY, Scott RM. Angiographic changes after pial synangiosis in childhood moyamoya disease. AJNR Am J Neuroradiol 1997;18:837-45.

7. Dusick JR, Gonzalez NR, Martin NA. Clinical and angiographic outcomes from indirect revascularization surgery for moyamoya disease in adults and children: A review of 63 procedures. Neurosurgery 2011;68:34-43.

8. Scott RM, Smith JL, Robertson RL, Madsen JR, Soriano SG, Rockoff MA. Long-term outcome in children with moyamoya syndrome after cranial revascularization by pial synangiosis. J Neurosurg 2004;100 2 Suppl Pediatrics:142-9.

9. Kawaguchi T, Fujita S, Hosoda K, Shose Y, Hamano S, Iwakura $\mathrm{M}$, et al. Multiple burr-hole operation for adult moyamoya disease. J Neurosurg 1996;84:468-76.

10. Sainte-Rose C, Oliveira R, Puget S, Beni-Adani L, Boddaert N, Thorne J, et al. Multiple bur hole surgery for the treatment of moyamoya disease in children. J Neurosurg 2006;105 6 Suppl: 437-43.

11. Oliveira RS, Amato MC, Simão GN, Abud DG, Avidago EB, Specian CM, et al. Effect of multiple cranial burr hole surgery on prevention of recurrent ischemic attacks in children with moyamoya disease. Neuropediatrics 2009;40:260-4.

12. Calviere L, Catalaa I, Marlats F, Januel AC, Lagarrigue J, Larrue V. Improvement in cognitive function and cerebral perfusion after bur hole surgery in an adult with moyamoya disease. Case report. J Neurosurg 2011;115:347-9.

13. Kapu R, Symss NP, Cugati G, Pande A, Vasudevan CM, Ramamurthi R. Multiple burr hole surgery as a treatment modality for pediatric moyamoya disease. J Pediatr Neurosci 2010;5:115-20.

14. Pandey P, Steinberg GK. Outcome of repeat revascularization surgery for moyamoya disease after an unsuccessful indirect revascularization. Clinical article. J Neurosurg 2011;115:328-36.

15. Zhao X, Wang C, Ji Y, Han C, Wang M. Therapeutic effect of multiple burr hole operation combined with dural inversion and periosteal synangiosis for moyamoya disease. $\mathrm{Br} \mathrm{J}$ Neurosurg 2015;29:811-7.

16. Ng J, Thompson D, Lumley JP, Saunders DE, Ganesan V. Surgical revascularisation for childhood moyamoya. Childs Nerv Syst 2012;28:1041-8.

17. Han C, Yang WZ, Zhang HT, Ye T, Duan L. Clinical characteristics and long-term outcomes of moyamoya syndrome associated with neurofibromatosis type 1. J Clin Neurosci 2015;22:286-90. 